\title{
Negative norm estimates and superconvergence results in Galerkin method for strongly nonlinear parabolic problems
}

\author{
Ambit Kumar Pany *, Morrakot Khebchareon ${ }^{\dagger}$, and Amiya K. Pani ${ }^{\ddagger}$
}

August 4, 2021

\begin{abstract}
The conforming finite element Galerkin method is applied to discretise in the spatial direction for a class of strongly nonlinear parabolic problems. Using elliptic projection of the associated linearised stationary problem with Gronwall type result, optimal error estimates are derived, when piecewise polynomials of degree $r \geq 1$ are used, which improve upon earlier results of Axelsson [Numer. Math. 28 (1977), pp. 1-14] requiring for $2 \mathrm{~d} r \geq 2$ and for $3 \mathrm{~d} r \geq 3$. Based on quasi-projection technique introduced by Douglas et al. [Math. Comp.32 (1978),pp. 345362], superconvergence result for the error between Galerkin approximation and approximation through quasi-projection is established for the semidiscrete Galerkin scheme. Further, a priori error estimates in Sobolev spaces of negative index are derived. Moreover, in a single space variable, nodal superconvergence results between the true solution and Galerkin approximation are established.

Keywords. Strongly nonlinear parabolic problems; Galerkin method; Elliptic projection; Global optimal error estimate; Quasi-projection; Negative norm estimate; Superconvergence.

AMS subject classifications. 65M15, 65N12, 65N30.
\end{abstract}

\section{Introduction}

In this paper, a priori error estimates with superconvergence results and negative norm estimates are derived for the conforming finite element Galerkin method applied to the following class of strongly nonlinear parabolic initial and boundary value problems: Find $u=u(x, t), x \in \Omega, t \in J=(0, T]$ for any $T>0$ satisfying

$$
\frac{\partial u}{\partial t}-\nabla \cdot A(u, \nabla u)+f(u, \nabla u)=0, \quad(x, t) \in \Omega \times J
$$

${ }^{*}$ Center of Applied Mathematics and Computation, SOA (Deemed to be University), Bhubaneswar-752030, India. Email: ambit.pany@gmail.com, ambitpany@soa.ac.in

${ }^{\dagger}$ Department of Mathematics, Faculty of Science, Chiang Mai University, Chiang Mai-50200 (Thailand) and Advanced Research Centre for Computational Simulation, Chiang Mai University, Chiang Mai-50200 (Thailand) and Centre of Excellence in Mathematics, CHE, 328 Si Ayutthaya Road, Bangkok, (Thailand). Email:morrakot.k@cmu.ac.th

${ }^{\ddagger}$ Department of Mathematics, Indian Institute of Technology Bombay, Powai, Mumbai-400076 (India). Email: akp@math.iitb.ac.in 
with initial and homogeneous Dirichlet boundary conditions

$$
\begin{array}{r}
u(x, 0)=u_{0}(x), \quad x \in \Omega, \\
u(x, t)=0, \quad x \in \partial \Omega, \quad t \in J,
\end{array}
$$

where $\Omega$ is a bounded domain in $\mathbb{R}^{d}, d=1,2,3$ with smooth boundary $\partial \Omega$. Further, $A=$ $\left(A_{1}, \cdots, A_{d}\right)$ and $f$ are vector and scalar valued functions defined on $\mathbb{R} \times \mathbb{R}^{d}$, respectively.

Throughout this article, we make the following assumptions called $\left(\mathbf{A}_{1}\right)$ on the coefficients:

(i) The problem (1.1)-(1.3) has a unique solution with bounded gradients $|\nabla u|,\left|\nabla u_{t}\right|$.

(ii) The function $A$ and $f$ are sufficiently smooth and bounded.

(iii) The matrix $\frac{\partial A}{\partial \xi}=\left[\frac{\partial A_{i}}{\partial \xi_{j}}\right]$, where $\xi=\left(\xi_{1}, \xi_{2}, \ldots, \xi_{d}\right)$ and $\xi_{j}=\frac{\partial u}{\partial x_{j}}$ is uniformly positive definite.

For existence, uniqueness and regularity results of such nonlinear equations, see [23]. For related numerical methods, a good number of article is devoted to strongly nonlinear elliptic problems, see, [4], 10], 14], [16], 17], 25], 28], 29], 35] and references, there in. However, there seems to be less number of papers available in literature on numerical approximation to strongly nonlinear parabolic problems, see, [3], [19], 30] and [22], etc. The more relevant article is [3], where conforming FEM is applied to the problem (1.1)-(1.3) and optimal error estimates in $L^{\infty}\left(L^{2}\right)$ are derived using piecewise polynomial of degree $r \geq 2$ for $d=2$ and for $d=3, r \geq 3$. One of our effort is to prove global optimal error estimates when $r \geq 1$.

Superconvergece, one of the objectives of this article, has long been an active area of research in finite element methods, see [33], [20], [21] and [9].This is mainly due to its applications in $a$ posteriori error estimates. One prominent line of thought is to post-process the discrete solution. The major tool used in this paper is an asymptotic expansion called quasi-projection of the approximate solution which leads to an expansion of the error. Essentially, being a postprocessing method, it is based on a sequence of projections associated with the approximate solution of the underlying stationary problem. Earlier, Douglas et al. [13] introduced quasi-projection technique for linear parabolic and second order hyperbolic equations and analyzed the superconvergence phenomena associated with $1 \mathrm{D}$-problems with the help of negative norm estimates. Later, Arnold and Douglas [2] generalized these results to a quasilinear parabolic equation of the form $c(x, t, u) u_{t}-\nabla \cdot[a(x, t, u) \nabla u+b(x, t, u)]+f(x, u, t)=0$. For similar results for parabolic integrodifferential equations, see, 27] and for the Stefan problem, see, [24]. For detailed survey of superconvergence result, refer to [20], 21] and [9] and references, there in. Regarding the application of negative norm estimates to prove interior superconvergence results is not new to the literature, see, [6] [5], and [31]. We strongly believe that the tools for global negative norm estimates will help to prove interior estimates and using the postprocessing technique in terms of certain averaging of operator applied to the Galerkin approximations,see [6], it is possible to prove interior superconvergence results, which will be a part of our future work.

The main contributions of this paper are summerised as follows. 
- Optimal error estimates of the semidiscrete Galerkin approximation are derived for the problem (1.1) in $d=1,2,3$.

Compared to Axelsson [3], additional superconvergence estimate in $L^{\infty}\left(H^{1}\right)$ for the error between the Galerkin approximation and the elliptic projection is proved and as a consequence, optimal estimates in $L^{\infty}\left(H^{1}\right)$ norm for $d=1,2,3$ and in $L^{\infty}\left(L^{\infty}\right)$-norm when $d=1,2$ are shown. The optimal error estimate in $L^{\infty}\left(L^{2}\right)$ is valid for $d=1,2,3$, when piecewise polynomials of degree $r \geq 1$ are used. But in [3], optimal estimate is valid for $r \geq 2$, that is, for quadratic or higher order elements in a one or two dimensional problems, where as in a three dimensional problems, optimal bound is derived for $r \geq 3$, that is, for cubic or higher order elements.

- Based on quasi-projection technique of Douglas et al. [13], superconvergence results are established for the error between Galerkin approximation and quasi-projection. As a result, optimal negative norm estimates are shown for the error between the semidiscrete Galerkin approximation and the exact solution.

- In a single space variable, knot superconvergence of semidiscrete Galerkin approximation to the solution of (1.1)-(1.3) is derived using quasi-projection technique of [13].

A general outline of the paper is as follows: In section 3, some basic result are derived. A quasi-projection is defined section 4 and section 5 deals with superconvergence phenomenon for a single space variable. The convergence at the knot points are shown to be of order $h^{2 r-\frac{1}{2}}$. Some of the results which are assumed in section 5 are proved in section 6 . In addition, an optimal $L^{\infty}$ estimate is also derived in this section.

\section{Some Notations and Preliminaries.}

In this section, we first recall the usual definitions of standard Sobolev spaces $W^{m, p}(\Omega)$ with the norm

$$
\|u\|_{m, p}:=\left(\sum_{|\alpha| \leq m}\left\|D^{\alpha} u\right\|^{p} d x\right)^{1 / p}, 1 \leq p<\infty
$$

and for $p=\infty$

$$
\|u\|_{m, \infty}:=\max _{|\alpha| \leq m}\left\|D^{\alpha} u\right\|_{L^{\infty}(\Omega)} .
$$

When $p=2$, denote $W^{m, 2}(\Omega)$ by $H^{m}(\Omega)$ with norm $\|\cdot\|_{m}=\|\cdot\|_{H^{m}(\Omega)}$. If $m=0$, then set $\|\cdot\|=\|\cdot\|_{L^{2}(\Omega)}$. Further, let $H_{0}^{1}=\left\{v \in H^{1}(\Omega): v=0 \quad\right.$ in $\left.\partial \Omega\right\}$ and let $H^{-s}$ be the dual space of $H^{s}$ with the norm

$$
\|v\|_{-s}=\sup _{w \in H^{s}\|w\|_{s} \neq 0} \frac{|<v, w>|}{\|w\|_{s}} .
$$

For a normed linear space $X$ with norm $\|\cdot\|_{X}$, let

$$
L^{p}(0, T ; X)=\left\{\phi:(0, T] \rightarrow X \text { with }\|\phi\|_{L^{p}(0, T ; X)}=\left[\int_{0}^{T}\|\phi(t)\|_{X}^{p} d t\right]^{\frac{1}{p}}<\infty\right\}
$$


and

$$
L^{\infty}(0, T ; X)=\left\{\phi:[0, T] \rightarrow X \mid\|\phi\|_{L^{\infty}(0, T ; X)}=\operatorname{esssup}_{0 \leq x \leq T}\|\phi(t)\|_{X}\right\} .
$$

The weak formulation of the problem (1.1) $-(1.3)$ is to find $u(t) \in H_{0}^{1}(\Omega)$ for $t \in(0, T]$ such that

$$
\left(u_{t}, v\right)+(A(u, \nabla u), \nabla v)+(f(u, \nabla u), v)=0, \quad v \in H_{0}^{1}(\Omega) .
$$

Throughout this paper, $C$ denotes a generic positive constant. By $C(q, k)$, we mean a generic constant depending on $\left\|\frac{\partial^{j} u}{\partial t^{j}}\right\|_{L^{\infty}\left(W^{q, \infty}\right)}$, for $j=0,1,2, \ldots, k$, but on no higher derivatives of $u$. For simplicity, we write $u_{t}$ for $\frac{\partial u}{\partial t}, A_{u}$ for $\frac{\partial A}{\partial u}$ and $A_{\xi}$ for $\frac{\partial A}{\partial \xi}$. Similarly for $f_{\xi}$ and $f_{u}$.

\section{Semidiscrete Galerkin Approximation}

This section deals with the semidiscrete formulation and elliptic projection with related error estimates.

Let $\mathcal{T}_{h}$ be a family of shape regular and quasi-uniform triangulation of $\bar{\Omega}$ into simplexes $K$. Let the discretization parameter $h$ be defined as $h=\max _{K \in \mathcal{T}_{h}} h_{K}$, where $h_{K}$ is the diameter of $K$. Further, let $\left\{V_{h}^{0}\right\}$ for $0<h \leq 1$ be a family of finite element subspace of $H_{0}^{1}$ defined by

$$
V_{h}^{0}=\left\{v \in C^{0}(\bar{\Omega}) \cap H_{0}^{1}:\left.\quad v\right|_{K} \in P_{r}(K) \quad \forall K \in \mathcal{T}_{h}\right\}
$$

where $P_{r}(K)$ is space of polynomials of degree less than equal to $r$. Note that $V_{h}^{0}$ for $0<h \leq 1$ satisfies the following approximation property: There exists a constant $C$ independent of $h$ such that for $\phi \in H^{q}(\Omega) \cap H_{0}^{1}$ with $q \in[1, r+1]$

$$
\inf _{\chi \in V_{h}^{0}}\left\{\|\phi-\chi\|+h\|\phi-\chi\|_{1}\right\} \leq C h^{q}\|\phi\|_{q} .
$$

In addition to (2.1), the following inverse assumption on $V_{h}^{0}$ holds for $v_{h} \in V_{h}^{0}$ and for $K \in \mathcal{T}_{h}$

$$
\left\|v_{h}\right\|_{H^{1}(K)} \leq C h_{K}^{-1}\left\|v_{h}\right\|_{L^{2}(K)} \quad \text { and } \quad\left\|v_{h}\right\|_{W^{j, \infty}(K)} \leq C h_{K}^{-\frac{d}{2}}\|v\|_{H^{j}(K)} j=0,1 .
$$

Note that for $1 \leq p \leq q \leq \infty$ and $\chi \in V_{h}^{0}$, the following property is valid:

$$
\|\chi\|_{L^{p}(K)} \leq C h_{K}^{d\left(\frac{1}{p}-\frac{1}{q}\right)}\|\chi\|_{L^{q}(K)} \quad \forall K \in \mathcal{T}_{h} .
$$

For properties (3.1)-(3.3), see [7].

The semidiscrete Galerkin approximation is now defined as a solution $U(t) \in V_{h}^{0}$ for $t \in J$ of

$$
\left(U_{t}, V\right)+(A(U, \nabla U), \nabla V)+(f(U, \nabla U), V)=0, V \in V_{h}^{0}
$$

with $U(0)=U_{0} \in V_{h}^{0}$ to be defined appropriately later on as an approximation of $u_{0}$.

For $u, v, W \in H_{0}^{1}(\Omega)$, define

$$
a(u ; W, v)=(A(W, \nabla W)-A(u, \nabla u), \nabla v)+(f(W, \nabla W)-f(u, \nabla u), v) .
$$


As in [3], it is easy to check that

$$
a(u, W ; W-u) \geq \rho\|\nabla(W-u)\|^{2}-\rho_{0}\|(W-u)\|^{2}, \quad u, W \in H_{0}^{1}(\Omega),
$$

where $\rho=\inf _{\left\{W, \nabla W \in \mathbb{R} \times \mathbb{R}^{n}\right\}}\left\{\right.$ smallest eigenvalues of $\left.\frac{\partial A}{\partial \xi}(W, \nabla W)\right\}>0$ and the constant $\rho_{0}=$ $\sup _{W, \nabla W}\left\{\frac{1}{2} \operatorname{div}\left[\frac{\partial A(W, \nabla W)}{\partial u}+\frac{\partial f(W, \nabla W)}{\partial u}\right]\right\}$.

Now using Taylor's expansion as in [3, we write

$$
\begin{aligned}
& a(u, W ; V)=b(u, \nabla u ; W-u, V) \\
& \quad+\int_{\Omega}\left[\nabla V^{T}, V\right] \int_{0}^{1}(1-s) \mathbb{A}^{\prime}(w, \nabla w) d s\left[\begin{array}{c}
\nabla(W-u) \\
W-u
\end{array}\right]\left[\begin{array}{c}
\nabla(W-u) \\
W-u
\end{array}\right] d x, \\
& \quad=b(u, \nabla u ; W-u, V)+\left(\mathbb{R}_{1}(W-u, \nabla(W-u)), \nabla V\right)+\left(\mathbb{R}_{2}(W-u, \nabla(W-u)), V\right),
\end{aligned}
$$

where $w=u+s(W-u), b(u, \nabla u ; \phi, \psi)=\int_{\Omega}\left[\nabla \psi^{T}, \psi\right] \mathbb{A}(u, \nabla u)\left[\begin{array}{c}\nabla \phi \\ \phi\end{array}\right] d x$,

$$
\mathbb{A}(w, \nabla w)=\left[\begin{array}{ll}
\frac{\partial A(w, \nabla w)}{\partial \xi}, & \frac{\partial A(w, \nabla w)}{\partial u} \\
\frac{\partial f(w, \nabla w)}{\partial \xi}, & \frac{\partial f(w, \nabla w)}{\partial u}
\end{array}\right],
$$

and $\mathbb{A}^{\prime}$ is the Fréchet derivative of $\mathbb{A}$. For similar expressions on reminder, one refers to [25] and [28]. The bilinear form $B(u, \nabla u ; \phi, \psi)$ associated with elliptic operator $\mathbb{L}$ is given by

$$
B(u, \nabla u ; \phi, \psi):=b(u, \nabla u ; \phi, \psi)+\lambda(\phi, \psi)=(\mathbb{L}(u) \phi, \psi),
$$

where

$$
\begin{aligned}
\mathbb{L}(u) \phi=- & \operatorname{div}\left(\frac{\partial A}{\partial \xi}(u, \nabla u) \nabla \phi\right)+\left[-\frac{\partial A}{\partial u}(u, \nabla u)+\frac{\partial f}{\partial \xi}(u, \nabla u)\right]^{T} \nabla \phi \\
+ & {\left[-\operatorname{div} \frac{\partial A}{\partial u}(u, \nabla u)+\frac{\partial f}{\partial u}(u, \nabla u)\right] \phi+\lambda \phi . }
\end{aligned}
$$

Here, $\lambda>0$ to be chosen large so that the bilinear form $B(u, \nabla u ; \cdot, \cdot)$ is coercive in the sense that

$$
B(\cdot, \cdot ; v, v) \geq \rho\|\nabla v\|^{2}+(\lambda-C(2,0))\|v\|^{2} \geq \alpha_{0}\|\nabla v\|^{2} .
$$

Note that $B(u, \nabla u ; \cdot, \cdot)$ is also bounded, that is, $|B(u, \nabla u ; v, w)| \leq C\|\nabla v\|\|\nabla w\|$. With $a_{\lambda}(u, W ; v)=$ $a(u, W ; v)+\lambda(W-u, v)$, it satisfies the following coercivity:

$$
a_{\lambda}(u, W ; W-u) \geq \alpha_{0}\|\nabla(W-u)\|^{2} .
$$

The Dirichlet problem for the bilinear form $B(u, \nabla u ; \cdot, \cdot)$ has a unique solution, see [11. Let $\mathbb{L}^{*}$ be the adjoint of $\mathbb{L}$ and $\psi \in H^{s}$ satisfies

$$
\begin{aligned}
\mathbb{L}^{*}(u) \phi & =\psi, \quad x \in \Omega, \\
\phi & =0, \quad x \in \partial \Omega,
\end{aligned}
$$


satisfying the following elliptic regularity, see [1]

$$
\|\phi\|_{s+2} \leq C(s+2,0)\|\psi\|_{s} .
$$

Let $\tilde{u}_{h}:[0, T] \rightarrow V_{h}^{0}$ be the elliptic projection of $u$ defined by

$$
B\left(u, \nabla u ; u-\tilde{u}_{h}, v\right)=0, \quad v \in V_{h}^{0} .
$$

For a given $u$, an application of the Lax-Milgram Lemma implies the existence of a unique $\tilde{u}_{h}$.

Let $\zeta=\tilde{u}_{h}-U, \eta=\tilde{u}_{h}-u$ and $e=U-u=\eta-\zeta$. Subtracting (2.1) from (3.4), and applying (3.7) with (3.12), we now arrive at

$$
\begin{aligned}
\left(\zeta_{t}, v\right)+ & B(u, \nabla u ; \zeta, v)=\left(\eta_{t}, v\right)+\lambda(\zeta, v)-\lambda(\eta, v) \\
& -\int_{\Omega}\left[\nabla v^{T}, v\right] \int_{0}^{1}(1-s) \mathbb{A}^{\prime}(w, \nabla w) d s\left[\begin{array}{c}
\nabla e \\
e
\end{array}\right]\left[\begin{array}{c}
\nabla e \\
e
\end{array}\right] d x \\
& =\left(\eta_{t}, v\right)+\lambda(\zeta, v)-\lambda(\eta, v)-\left(\mathbb{R}_{1}(e, \nabla e), \nabla v\right)+\left(\mathbb{R}_{2}(e, \nabla e), v\right),
\end{aligned}
$$

where $w=u+s(U-u)=u+s e$.

\subsection{Elliptic projection.}

This subsection deals with an elliptic projection as defined by Wheeler [34] and related error analysis.

We need for our subsequent use, the interaction of $B(u, \nabla u ; \cdot, \cdot)$ with time differentiation, when the coefficient of $\mathbb{L}$ associated with this bilinear form are time dependent. Now, let $\phi: J \rightarrow H_{0}^{1}$ and $\psi \in H_{0}^{1} \cap H^{s+2}$. Then, a simple use of the Leibnitz's rule yields

$$
\begin{aligned}
\frac{d^{k}}{d t^{k}} B(u, \nabla u ; \phi, \psi)= & \sum_{i=0}^{k}\left(\begin{array}{c}
k \\
i
\end{array}\right) \int_{\Omega}\left[\nabla \psi^{T}, \psi\right]\left[\frac{d^{k-i}}{d t^{k-i}} \mathbb{A}(u, \nabla u)\right] \frac{d^{i}}{d t^{i}}\left[\begin{array}{c}
\nabla \phi \\
\phi
\end{array}\right] d x \\
& +\lambda\left(\frac{\partial^{k} \phi}{\partial t^{k}}, \psi\right) \\
= & B\left(u, \nabla u ; \frac{\partial^{k} \phi}{\partial t^{k}}, \psi\right)+\sum_{i=0}^{k-1} F_{i k}\left(u, \nabla u ; \frac{\partial^{i} \phi}{\partial t^{i}}, \psi\right)
\end{aligned}
$$

where for $\gamma \in H_{0}^{1}$,

$$
\begin{aligned}
F_{i k}(u, \nabla u ; \gamma, \psi) & =\left(\begin{array}{c}
k \\
i
\end{array}\right) \int_{\Omega}\left[\nabla \psi^{T}, \psi\right]\left[\frac{d^{k-i}}{d t^{k-i}} \mathbb{A}(u, \nabla u)\right]\left|\begin{array}{c}
\nabla \gamma \\
\gamma
\end{array}\right| d x \\
& =\left(\begin{array}{c}
k \\
i
\end{array}\right)\left(\gamma,-\nabla\left(\frac{d^{k-i}}{d t^{k-i}}\right)\left(\frac{\partial A}{\partial \xi}\right) \nabla \psi+\left(\frac{d^{k-i}}{d t^{k-i}}\right)\left(\frac{\partial f}{\partial \xi}\right) \psi\right) \\
& +\left(\frac{d^{k-i}}{d t^{k-i}}\right)\left(\frac{\partial A}{\partial u}\right) \nabla \psi^{T}+\left(\frac{d^{k-i}}{d t^{k-i}}\right)\left(\frac{\partial f}{\partial u}\right) \psi
\end{aligned}
$$


Therefore,

$$
\left|F_{i k}(u, \nabla u ; \phi, \psi)\right|=\left\{\begin{array}{l}
C(1, k-i)\|\phi\|_{1}\|\psi\|_{1}, \\
C(s+2, k-i)\|\phi\|_{-s}\|\psi\|_{s+2}, \quad s=0,1,2, \ldots
\end{array}\right.
$$

For convenience, we prove the following lemma, we refer [2] for an analogous result.

Lemma 3.1 Let there be given a linear functional $F: H_{0}^{1} \rightarrow \mathbb{R}$ and numbers $M_{1} \geq M_{2} \geq M_{3} \geq$ $\ldots \geq M_{p+1}, 0 \leq p \leq r$ with

$$
|F(\rho)| \leq M_{s+2}\|\rho\|_{s+2} \text { for } \rho \in H^{s+2}(\Omega) \cap H_{0}^{1}(\Omega), s=-1,0,1, \ldots, p-1 .
$$

Suppose $\varphi \in H_{0}^{1}(\Omega)$ satisfies

$$
B(u, \nabla u ; \varphi, \chi)=F(\chi) \quad \text { for } \quad \chi \in V_{h}^{0},
$$

then,

$$
\begin{array}{r}
\|\varphi\|_{-s} \leq C(\max (s, 0)+2,0)\left[\left(M_{1}+\inf _{\chi \in V_{h}^{0}}\|\varphi-\chi\|_{1}\right) h^{s+1}+M_{s+2}\right] \\
s=-1,0,1, \ldots, p-1 .
\end{array}
$$

Proof. For $s=-1$, we note that

$$
\begin{aligned}
B(u, \nabla u ; \varphi, \varphi) & =[B(u, \nabla u ; \varphi, \varphi-\chi)+F(\chi-\varphi)+F(\varphi)], \quad \chi \in M_{h}^{0} \\
& \leq\left(C\|\varphi\|_{1}+M_{1}\right) \inf _{\chi \in V_{h}^{0}}\|\varphi-\chi\|_{1}+M_{1}\|\varphi\|_{1} .
\end{aligned}
$$

Since $B(u, \nabla u ; \cdot, \cdot)$ is coercive, it now follows that

$$
\|\varphi\|_{1} \leq C(2,0)\left(M_{1}+\inf _{\chi \in V_{h}^{0}}\|\varphi-\chi\|\right),
$$

which yields the desired for $s=-1$.

For $s=0,1,2, \ldots, p-1$, we apply Aubin-Nitsche's duality argument, Given $\psi \in H^{s}(\Omega), 0 \leq$ $s \leq p-1$, define $\phi \in H^{s+2}(\Omega) \cap H_{0}^{1}(\Omega)$ by (3.9)-(3.10). Now, a use of boundedness of the bilinear form with bound for $F$, approximation property (3.1) and (3.11) yields

$$
\begin{aligned}
(\varphi, \psi) & =\left(\varphi, \mathbb{L}^{*}(u) \phi\right)=B(u, \nabla u ; \varphi, \phi) \\
& =B(u, \nabla u ; \varphi, \phi-\chi)+F(\chi-\phi)+F(\phi), \quad \chi \in V_{h}^{0} \\
& \leq C(s+2,0)\left(\|\varphi\|_{1}+M_{1}\right) \inf _{\chi \in V_{h}^{0}}\|\phi-\chi\|_{1}+M_{s+2}\|\phi\|_{s+2} \\
& \leq C(s+2,0)\left(\|\varphi\|_{1}+M_{1}\right) h^{s+1}\|\phi\|_{s+2}+M_{s+2}\|\phi\|_{s+2} \\
& \leq C(s+2,0)\left(\left[\|\varphi\|_{1}+M_{1}\right] h^{s+1}+M_{s+2}\right)\|\psi\|_{s} .
\end{aligned}
$$


For $s=0$, we obtain

$$
\left.\|\varphi\| \leq C(2,0)\left[\left(\|\varphi\|_{1}+M_{1}\right) h+M_{2}\right)\right],
$$

which completes the desired result for $s=0$. The other negative estimate follows similarly and this concludes the rest of the proof.

Below, we discuss the negative norm estimate for $\eta_{t}$.

Theorem 3.1 Let $1 \leq q \leq r+1$ and $\frac{\partial^{k} u}{\partial t^{k}} \in H^{q}(\Omega)$, for $t \in J$. Then, it holds

$$
\left\|\frac{\partial^{k} \eta}{\partial t^{k}}\right\|_{-s} \leq C(\max (q, s+2), k) h^{s+q},-1 \leq s \leq r-1 .
$$

Proof. A use of (3.14) shows

$$
B\left(u, \nabla u ; \frac{\partial^{k} \eta}{\partial t^{k}}, \chi\right)=-\sum_{i=0}^{k-1} F_{i k}\left(u, \nabla u ; \frac{\partial^{i} \eta}{\partial t^{i}}, \chi\right), \quad \chi \in V_{h}^{0} .
$$

Now, we identify the right hand side as $F_{K}(\chi)$. Then, for $k=0, F_{K}=0$, an application of the Lemma 3.1 and

$$
\inf _{\chi \in V_{h}^{0}}\|\eta-\chi\|_{1}=\inf _{\chi \in V_{h}^{0}}\|u-\chi\|_{1} \leq C(q, 0) h^{q-1}
$$

shows the result for $k=0$.

For the general case, we resort to induction on $k$. Let the assertion of the theorem be true for $k-1$. Then, we claim that conclusion also holds for $k$. For all $\rho \in H^{s}(\Omega) \cap H_{0}^{1}(\Omega)$, we arrive from (3.15) at

$$
\begin{aligned}
\left|F_{i k}\left(u, \nabla u ; \frac{\partial^{i} \eta}{\partial t^{i}}, \rho\right)\right| & \leq C(\max (s, 0)+2, k) \sum_{i=0}^{k-1}\left\|\frac{\partial^{i} \eta}{\partial t^{i}}\right\|_{-s}\|\rho\|_{s+2} \\
& \leq C(\max (q, s+2), k) h^{s+q}\|\rho\|_{s+2}, \quad s=-1,0,1, \ldots, r-1 .
\end{aligned}
$$

Here, we have used the induction hypothesis to derive the second inequality. Since,

$$
\inf _{\chi \in V_{h}^{0}}\left\|\frac{\partial^{k} \eta}{\partial t^{k}}-\chi\right\|_{1}=\inf _{\chi \in V_{h}^{0}}\left\|\frac{\partial^{k} u}{\partial t^{k}}-\chi\right\|_{1} \leq C(q, k) h^{q-1},
$$

the use of lemma 3.1 completes the rest of the proof.

Moreover, following standard argument for linear elliptic problems, see Brenner and Scott [7], the following $L^{p}$ for $1 \leq p \leq \infty$ estimate holds for both $\eta$ and $\eta_{t}$ and for $1 \leq q \leq r+1$

$$
\|\eta\|_{L^{p}}+h\|\nabla \eta\|_{L^{p}} \leq C h^{q}\|u(t)\|_{W^{r+1, p}} \text { and }\left\|\eta_{t}(t)\right\|_{L^{p}} \leq C h^{r+1}\left\|u_{t}(t)\right\|_{W^{r+1, p}} .
$$

\section{Global Error Estimates}

This section focusses on the optimal error estimates of $u-U$ in $L^{\infty}\left(L^{2}\right)$ and $L^{\infty}\left(H^{1}\right)$-norms.

The following two Lemma shows both $L^{\infty}\left(L^{2}\right)$ and $L^{\infty}\left(H^{1}\right)$ error estimation for $\zeta=U-\tilde{u}_{h}$. 
Lemma 4.1 Let $2 \leq q \leq r+1$. Then, the following estimate

$$
\|\zeta\|_{L^{\infty}\left(L^{2}\right)}+\alpha_{0}\|\zeta\|_{L^{2}\left(H^{1}\right)} \leq C(\max (q, 2 k+1), k)\left(h^{q}+h^{2(q-1)}\right)
$$

holds.

Proof. From (3.13), we now rewrite it as

$$
\begin{aligned}
\left(\zeta_{t}, v\right)+a_{\lambda}\left(\tilde{u}_{h}, U ; v\right)=( & \left.\eta_{t}, v\right)+\lambda(\zeta, v)-\lambda(\eta, v) \\
& +\left(\mathbb{R}_{1}(\eta, \nabla \eta), \nabla v\right)+\left(\mathbb{R}_{2}(\eta, \nabla \eta), v\right) .
\end{aligned}
$$

Setting $v=\zeta$ in (4.2), a use of coercivity of $a_{\lambda}\left(\tilde{u}_{h}, U ; \zeta\right)$ with the Cauchy-Schwartz inequality yields

$$
\begin{aligned}
\frac{1}{2} \frac{d}{d t}\|\zeta(t)\|^{2}+\alpha_{0}\|\nabla \zeta(t)\|^{2} \leq( & \left.\left\|\eta_{t}\right\|_{-1}+\lambda\|\eta\|_{-1}\right)\|\nabla \zeta\|+\lambda\|\zeta\|^{2} \\
& +\left|\left(\mathbb{R}_{1}(\eta, \nabla \eta), \nabla \zeta\right)\right|+\left|\left(\mathbb{R}_{2}(\eta, \nabla \eta), \zeta\right)\right|
\end{aligned}
$$

For the last two terms on the right hand side of (4.3) , we use the form of $\mathbb{R}_{1}$ and $\mathbb{R}_{2}$ and generalized Hölder inequality with $L^{4}$ estimate (3.20) to obtain

$$
\begin{aligned}
\left|\left(\mathbb{R}_{1}(\eta, \nabla \eta), \nabla \zeta\right)\right| & \leq C\left(\|\eta\|_{L^{4}}^{2}+\|\nabla \eta\|_{L^{4}}^{2}\right)\|\nabla \zeta\| \\
& \leq C h^{2(q-1)}\|\nabla \zeta\|,
\end{aligned}
$$

and similarly, we arrive at

$$
\left|\left(\mathbb{R}_{2}(\eta, \nabla \eta), \zeta\right)\right| \leq C h^{2(q-1)}\|\zeta\|
$$

On substitution in (4.3), a use of Young's inequality with kickback argument and application of Gronwall's Lemma completes the rest of the proof.

Our next Lemma concerns with estimate of $\zeta$ in gradient norm.

Lemma 4.2 Let $\max ((1+d / 2), 2) \leq q \leq r+1$. Then, the following estimate holds

$$
\left\|\zeta_{t}\right\|_{L^{2}\left(L^{2}\right)}+\alpha_{0}\|\zeta\|_{L^{\infty}\left(H^{1}\right)} \leq C(\max (q, 2 k+2), k) h^{q} .
$$

Proof. Choosing $v=\zeta_{t}$ in (3.13), we arrive at

$$
\begin{aligned}
\left\|\zeta_{t}\right\|^{2}+B\left(\tilde{u}_{h}, \nabla \tilde{u}_{h} ; \zeta, \zeta_{t}\right) & =\left(\eta_{t}, \zeta_{t}\right)+\lambda\left(\zeta, \zeta_{t}\right)-\lambda\left(\eta, \zeta_{t}\right) \\
& +\left(\mathbb{R}_{1}(e, \nabla e), \nabla \zeta_{t}\right)+\left(\mathbb{R}_{2}(e, \nabla e), \zeta_{t}\right) .
\end{aligned}
$$

Note that from (3.14) with $k=1$, we obtain

$$
B\left(\tilde{u}_{h}, \nabla \tilde{u}_{h} ; \zeta, \zeta_{t}\right):=\frac{1}{2} \frac{d}{d t} B\left(\tilde{u}_{h}, \nabla \tilde{u}_{h} ; \zeta, \zeta\right)-F_{01}\left(\tilde{u}_{h}, \nabla \tilde{u}_{h} ; \zeta, \zeta\right)
$$

On substitution (4.8) in (4.7), it follows that

$$
\begin{aligned}
\left\|\zeta_{t}\right\|^{2}+\frac{1}{2} \frac{d}{d t} B\left(\tilde{u}_{h}, \nabla \tilde{u}_{h} ; \zeta, \zeta\right) & =\left(\left(\eta_{t}, \zeta_{t}\right)+\lambda\left(\zeta, \zeta_{t}\right)-\lambda\left(\eta, \zeta_{t}\right)\right)+F_{01}\left(\tilde{u}_{h}, \nabla \tilde{u}_{h} ; \zeta, \zeta\right) \\
& +\left(\mathbb{R}_{1}(e, \nabla e), \nabla \zeta_{t}\right)+\left(\mathbb{R}_{2}(e, \nabla e), \zeta_{t}\right) \\
& =I_{1}\left(\zeta_{t}\right)+I_{2}\left(\zeta_{t}\right)+I_{3}\left(\zeta_{t}\right)+I_{4}\left(\zeta_{t}\right) .
\end{aligned}
$$


For the first term on the right hand side of (4.9), apply the Cauchy-Schwarz inequality, estimates of $\eta$ and $\eta_{t}$ with estimate from Lemma 4.1 to find that

$$
\begin{aligned}
\left|I_{1}\left(\zeta_{t}\right)\right| & \leq\left(\left\|\eta_{t}\right\|+\lambda\|\eta\|+\lambda\|\zeta\|\right)\left\|\zeta_{t}\right\| \\
& \leq C h^{2 q}+\frac{1}{8}\left\|\zeta_{t}\right\|^{2} .
\end{aligned}
$$

For the second term on the right hand side of (4.9), a use of (3.15) with $i=0, k=1$ and the stability of the elliptic projection in $H^{1}$ yields

$$
\left|I_{2}\left(\zeta_{t}\right)\right| \leq C(1,1)\|\nabla \zeta\|^{2}
$$

In order to estimate the fourth term on the right hand side of (4.9), we observe using definition of $\mathbb{R}_{2}$, the generalized Hölder's inequality, the Sobolev inequality, inverse inequality and the Young's inequality that

$$
\begin{aligned}
\left|I_{4}\left(\zeta_{t}\right)\right| & \leq C \int_{\Omega}\left(|e|^{2}+|e||\nabla e|+|\nabla e|^{2}\right)\left|\zeta_{t}\right| d x \\
& \leq C\left(\|e\|_{L^{4}}^{2}+\|\nabla e\|_{L^{4}}^{2}\right)\left\|\zeta_{t}\right\| \\
& \leq C\left(h^{2 q}+h^{-d / 2}\|\zeta\|^{2}+h^{2(q-1)}+h^{-d / 2}\|\nabla \zeta\|^{2}\right)\left\|\zeta_{t}\right\| \\
& \leq C\left(h^{4(q-1)-d}+h^{-d}\|\nabla \zeta\|^{4}\right)+\frac{1}{8}\left\|\zeta_{t}\right\|^{2} .
\end{aligned}
$$

For the estimate of $I_{3}\left(\zeta_{t}\right)$, rewrite it as

$$
I_{3}\left(\zeta_{t}\right)=\frac{d}{d t}\left(\mathbb{R}_{1}(e, \nabla e), \nabla \zeta\right)-\left(\frac{d}{d t} \mathbb{R}_{1}(e, \nabla e), \nabla \zeta\right)
$$

Note that using the definition of $\mathbb{R}_{1}(e, \nabla e)$ and the chain rule, an application of generalized Hölder's inequality with inverse inequality and maximum norm bounds of $\eta$ and $\nabla \eta$ yields

$$
-\left(\frac{d}{d t} \mathbb{R}_{1}(e, \nabla e), \nabla \zeta\right):=-\left(\mathbb{R}_{1}(e, \nabla e)^{\prime}, \nabla \zeta\right)-\left(\mathbb{R}_{1, t}(e, \nabla e), \nabla \zeta\right),
$$

where $\mathbb{R}_{1}(e, \nabla e)^{\prime}$ is the time derivative of the variable $e$ and $\nabla e$ using chain rule and $\mathbb{R}_{1, t}(e, \nabla e)$ is the time derivative of the coefficients again using chain rule. For the estimate of the first term on the right hand side of (4.14), we obtain

$$
\begin{aligned}
-\left(\mathbb{R}_{1}(e, \nabla e)^{\prime}, \nabla \zeta\right) & \leq C \int_{\Omega}\left(\left|e_{t}\right|+\left|\nabla e_{t}\right|\right)(|e|+|\nabla e|)|\nabla \zeta| d x \\
& \leq C\left\|\nabla e_{t}\right\|\|\nabla e\|_{L^{\infty}}\|\nabla \zeta\| \\
& \leq C\left(h^{q-1}+h^{-1}\left\|\zeta_{t}\right\|\right)\left(h^{q-1}+h^{-d / 2}\|\nabla \zeta\|\right)\|\nabla \zeta\| \\
& \leq C\left(\left(h^{4(q-1)}+h^{2 q}\right)+\|\nabla \zeta\|^{2}+h^{-2(1+d / 2)}\|\nabla \zeta\|^{4}\right)+\frac{1}{8}\left\|\zeta_{t}\right\|^{2} .
\end{aligned}
$$


For the second term on the right hand side of (4.14), we bound it as

$$
\begin{aligned}
-\left(\mathbb{R}_{1, t}(e, \nabla e), \nabla \zeta\right) & \leq C \int_{\Omega}\left(1+\left|e_{t}\right|+\left|\nabla e_{t}\right|\right)\left(|e|^{2}+|e \| \nabla e|+|\nabla e|^{2}\right)|\nabla \zeta| d x \\
& \leq C\left(\|e\|_{L^{4}}^{2}+\|\nabla e\|_{L^{4}}^{2}\right)\|\nabla \zeta\|+C\left(\left\|e_{t}\right\|+\left\|\nabla e_{t}\right\|\right)\left(\|e\|_{L^{\infty}}^{2}+\|\nabla e\|_{L^{\infty}}\right)\|\nabla \zeta\| \\
& \left.\leq C\left(\|\nabla e\|^{2}+\|\nabla e\|_{L^{4}}^{2}\right)\|\nabla \zeta\|+C\left\|\nabla e_{t}\right\|\|\nabla e\|_{L^{\infty}}\right)\|\nabla \zeta\| \\
& \leq C\left(h^{2(q-1)}+\left(1+h^{-d / 2}\right)\|\nabla \zeta\|^{2}\right)\|\nabla \zeta\| \\
& +C\left(h^{q-1}+h^{-1}\left\|\zeta_{t}\right\|\right)\left(h^{q-1}+h^{-d / 2}\|\nabla \zeta\|\right)\|\nabla \zeta\| \\
& \leq C\left(\left(h^{4(q-1)}+h^{2 q}\right)+\|\nabla \zeta\|^{2}+h^{-2(1+d / 2)}\|\nabla \zeta\|^{4}\right)+\frac{1}{8}\left\|\zeta_{t}\right\|^{2} .
\end{aligned}
$$

On substitution of the estimates (4.10)-(4.13) in (4.9), an integration with respect to time with $\zeta(0)=0$, (4.4), the Young's inequality, kickback arguments and estimate of $\eta$ and its gradient yields

$$
\begin{aligned}
\int_{0}^{t}\left\|\zeta_{t}(s)\right\|^{2} d s+\alpha_{0}\|\nabla \zeta(t)\|^{2} & \leq C(\max (q, 2 k+1), k)\left(h^{2 q}+\int_{0}^{t}\left(h^{-2(1+d / 2)}\|\nabla \zeta\|^{4}\right.\right. \\
& \left.\left.+\left(1+h^{4(q-1)-2}\right)\|\nabla \zeta\|^{2}\right) d s\right) .
\end{aligned}
$$

An application of Gronwall's inequality with use of $L^{2}\left(L^{2}\right)$ estimate of $\nabla \zeta$ and for $(1+d / 2) \leq q \leq$ $r+1$ shows the result and this completes the rest of the proof.

A use of triangle inequality with estimates of Lemmas $4.1+4.2$ and Theorem 3.1 yields easily the following result.

Theorem 4.1 Let $e=U-u$, where $u$ is the solution of (3.1) and $U$ is the solution of (3.2) with $U(0)$ defined as in (4.3), for some $k$ such that $\max ((1+d / 2), 2) \leq q \leq r+1$

$$
\|e\|_{L^{\infty}\left(L^{2}\right)}+h\left(\|e\|_{L^{2}\left(H^{1}\right)}+\|e\|_{L^{\infty}\left(H^{1}\right)}\right) \leq C(\max (q, 2 k+2), \max (k, 1)) h^{q} .
$$

Remark 4.1 Note that using Lemma 4.1 and Theorem 3.1 with triangle inequality, we obtain optimal in $\|U-u\|_{L^{\infty}\left(L^{2}\right)}$ - estimate, when $2 \leq q \leq r+1$, that is, for $r \geq 1$.

Now, we obtain the following corollary.

Corollary 4.1 Under the assumptions of Lemma 4.2, there holds for $\max ((1+d / 2), 2) \leq q \leq r+1$

$$
\left\|\mathbb{R}_{2}\right\|_{L^{2}\left(L^{2}\right)}+h\left\|\mathbb{R}_{1}\right\|_{L^{2}\left(L^{2}\right)} \leq C(\max (q, 2 k+2))\left(h^{2(q-1)}+h^{(2 q-d / 2)}\right) .
$$

Proof. From the definition of $\mathbb{R}_{2}$, a use of estimate of $\eta$ from (3.20) with (4.6) and inverse estimate 
yields

$$
\begin{aligned}
\left\|\mathbb{R}_{2}\right\|_{L^{2}\left(L^{2}\right)}=\sup _{\|v\|_{L^{2}\left(L^{2}\right)}=1}\left|\int_{0}^{T}\left(\mathbb{R}_{2}, v\right) d s\right| & \leq C\left(\int_{0}^{T} \int_{\Omega}\left(|e|^{4}+|e|^{2}|\nabla e|^{2}+|\nabla e|^{4}\right) d x d s\right)^{1 / 2} \\
& \leq C \int_{0}^{T}\left(\|e\|_{L^{4}}^{2}+\|\nabla e\|_{L^{4}}^{2}\right) d s \\
& \leq C \int_{0}^{T}\left(h^{2 q}+\|\zeta\|_{L^{4}}^{2}+h^{2(q-1)}+h^{-d / 2}\|\nabla \zeta\|^{2}\right) d s \\
& \leq C(T)\left(h^{2 q}+\|\nabla \zeta\|_{L^{2}\left(L^{2}\right)}^{2}+h^{2(q-1)}+h^{-d / 2}\|\nabla \zeta\|_{L^{2}\left(L^{2}\right)}^{2}\right) \\
& \leq C\left(h^{2(q-1)}+h^{2 q-d / 2}\right) .
\end{aligned}
$$

Similarly, the estimate

$$
\left\|\mathbb{R}_{1}\right\|_{L^{2}\left(H^{-1}\right)} \leq C\left(h^{2(q-1)}+h^{2 q-d / 2}\right)
$$

holds and the rest of the proof follows.

As a consequence of superconvergence result of $\|\nabla \zeta\|$ from (4.6), the following maximum norm estimate is derived which we put as a Theorem.

Theorem 4.2 There holds for $2 \leq q \leq r+1$

$$
\|U-u\|_{L^{\infty}\left(L^{\infty}\right)} \leq C(\max (q, 2 k+2), \max (k, 1))(\log (1 / h))^{m} h^{q},
$$

where $m=0$ for $d=1$, and $m=1$ when $d=2$.

Proof. From the superconvergence result (4.6), we obtain for $d=1$, the maximum norm estimate of $\zeta$, that is,

$$
\|\zeta\|_{L^{\infty}\left(L^{\infty}\right)} \leq\|\zeta\|_{L^{\infty}\left(H^{1}\right)} \leq C(\max (q, 2 k+2), \max (k, 1)) h^{q} .
$$

Therefore, a use of triangle inequality with (4.23) and (3.20) for $p=\infty$ yields for 1D-problem the following max norm estimate of $e$ for $2 \leq q \leq r+1$

$$
\|U-u\|_{L^{\infty}\left(L^{\infty}\right)} \leq C(\max (q, 2 k+2), \max (k, 1)) h^{q} .
$$

For $d=2$, using Sobolev inequality, we arrive from the superconvergence result (4.6), the max norm estimate

$$
\|\zeta\|_{L^{\infty}\left(L^{\infty}\right)} \leq C \log (1 / h)\|\zeta\|_{L^{\infty}\left(H^{1}\right)} \leq C(\max (q, 2 k+2), \max (k, 1)) \log (1 / h) h^{q}
$$

Hence, we derive the max norm estimate for $2 \mathrm{D}$-problem for $2 \leq q \leq r+1$ as

$$
\|U-u\|_{L^{\infty}\left(L^{\infty}\right)} \leq C(\max (q, 2 k+2), \max (k, 1)) \log (1 / h) h^{q} .
$$

This completes the rest of the proof. 


\section{The Quasi-projection}

Let $z_{0}=\eta$ and $\theta_{0}=\zeta$. Define maps $z_{j}: J \rightarrow V_{h}^{0}$ recursively by

$$
B\left(u, \nabla u ; z_{j}, v\right)=-\left(\frac{\partial z_{j-1}}{\partial t}, v\right), \quad v \in V_{h}^{0}, \quad t \in J, \quad j=1,2, \ldots
$$

Theorem 5.1 Let $j \geq 0, k \geq 0,1 \leq q \leq r+1$ and assume that $\frac{\partial^{j+k} u}{\partial t^{j+k}} \in H^{q}(\Omega)$ for $t \in J$. Then for $-1 \leq s \leq r-1-2 j$, the following estimate holds

$$
\left\|\frac{\partial^{k} z_{j}}{\partial t^{k}}\right\|_{-s}=C(\max (q, \max (s, 0)+2 j+2), k+j) h^{s+q+2 j} .
$$

Proof. The proof is carried out by induction $j$. For $j=0, z_{0}=\eta$ and this case is covered by Theorem 3.1. Now suppose that (5.2) is true for $j-1$, then we show (5.2) to be true for $j$. For $j>0$ and $k \geq 0$, let

$$
F(\rho)=-\sum_{i=0}^{k-1} F_{i k}\left(\frac{\partial^{i} z_{j}}{\partial t^{i}}, \rho\right)-\left(\frac{d^{k}}{d t^{k}}\left(\frac{\partial z_{j-1}}{\partial t}\right), \rho\right), \quad \rho \in H^{s+2}(\Omega) \cap H_{0}^{1}(\Omega) .
$$

Then,

$$
B\left(u, \nabla u ; \frac{\partial^{k} z_{j}}{\partial t^{k}}, \chi\right)=F(\chi), \quad \chi \in V_{h}^{0}+H^{s+2}(\Omega) .
$$

Now

$$
\begin{aligned}
|F(\rho)| & \leq\left|\sum_{i=0}^{k-1} F_{i k}\left(\frac{\partial^{i} z_{j}}{\partial t^{i}}, \rho\right)\right|+\left|\left(\frac{d^{k}}{d t^{k}}\left(\frac{\partial z_{j-1}}{\partial t}\right), \rho\right)\right| \\
& \leq C(\max (s, 0)+2, k) \sum_{i=0}^{k-1}\left\|\frac{\partial^{i} z_{j}}{\partial t^{i}}\right\|_{-s}\|\rho\|_{s+2}+\left|\left(\frac{\partial^{k+1}}{\partial t^{k+1}} z_{j-1}, \rho\right)\right| \\
& \leq\left\{C(\max (s, 0)+2, k) \sum_{i=0}^{k-1}\left\|\frac{\partial^{i} z_{j}}{\partial t^{i}}\right\|_{-s}+\left\|\frac{\partial^{k+1}}{\partial t^{k+1}} z_{j-1}\right\|_{-s-2}\right\}\|\rho\|_{s+2} \\
& \leq\left\{C(\max (s, 0)+2, k) \sum_{i=0}^{k-1}\left\|\frac{\partial^{i} z_{j}}{\partial t^{j}}\right\|_{-s}+C(\max (q, \max (s, 0)+2 j+2), k+j) h^{s+q+2 j}\right\}\|\rho\|_{s+2} .
\end{aligned}
$$

The last inequality uses induction hypothesis. If we consider $p=r-2 j$

$$
M_{s+2}=C(\max (q, \max (s, 0)+2 j+2), k+j)\left(h^{s+q+2 j}+\sum_{i=0}^{k-1}\left\|\frac{\partial^{i} z_{j}}{\partial t^{i}}\right\|_{-s}\right)
$$

for $s=-1,0,1, \ldots, r-2 j-1$, in the Lemma 3.1, then $F$ fulfills all the hypothesis. Therefore, we obtain

$$
\left\|\frac{\partial^{k} z_{j}}{\partial t^{k}}\right\|_{-s} \leq C(\max (s, 0)+2,0)\left[\left(M_{1}+\inf _{\chi \in V_{h}^{0}}\left\|\frac{\partial^{k} z_{j}}{\partial t^{k}}-\chi\right\|\right) h^{s+1}+M_{s+2}\right]
$$


for $s=-1,0, \ldots, r-2 j-1$. As $z_{j} \in V_{1}^{0}$, the infimum appearing here is zero and it follows that

$$
\begin{aligned}
\left\|\frac{\partial^{k} z_{j}}{\partial t^{k}}\right\|_{-s} & \leq C(\max (q, \max (s, 0)+2 j+2), k+j)\left[h^{s+q+2 j}\right. \\
& \left.+\sum_{i=0}^{k-1}\left\|\frac{\partial^{i} z_{j}}{\partial t^{i}}\right\|_{1} h^{s+1}+\sum_{i=0}^{k-1}\left\|\frac{\partial^{i} z_{j}}{\partial t^{i}}\right\|_{-s}\right] .
\end{aligned}
$$

For $k=0$, the inequality (5.2) follows. For $k \geq 1$, the theorem can be completed by simple induction on $k$. This completes the induction on $j$ and hence, the inequality (5.2) is proved. This completes the rest of the proof.

For one of the main superconvergent result, we can choose the initial condition $U$ at $t=0$ as

$$
U(0)=\tilde{u}(0)+z_{1}(0)+\ldots+z_{k}(0), \quad \text { for } 2 k \leq r-1 .
$$

Let $\theta_{k}=\zeta+z_{1}+\ldots+z_{k}$. Then for all $v \in V_{h}^{0}$,

$$
\begin{aligned}
\left(\frac{\partial \theta_{k}}{\partial t}, v\right)+B\left(u, \nabla u ; \theta_{k}, v\right)= & \left(\frac{\partial z_{k}}{\partial t}, v\right)-\lambda\left(z_{k}, v\right)+\lambda\left(\theta_{k}, v\right) \\
& +\left(\mathbb{R}_{1}(e, \nabla e), \nabla v\right)+\left(\mathbb{R}_{2}(e, \nabla e, v),\right.
\end{aligned}
$$

where

$$
\left(\mathbb{R}_{1}(e, \nabla e), \nabla v\right)+\left(\mathbb{R}_{2}(e, \nabla e), v\right)=-\int_{\Omega}\left[\nabla v^{T}, v\right] \int_{0}^{1}(1-s) \mathbb{A}^{\prime}(w, \nabla w) d s\left[\begin{array}{c}
\nabla e \\
e
\end{array}\right]\left[\begin{array}{c}
\nabla e \\
e
\end{array}\right] d x
$$

with $\theta_{k}(0)=0$. The above relation follows as directed consequence of (3.13) and the definition of the $z_{j}$ 's.

The following theorem gives a bound for $\theta_{k}$.

Theorem 5.2 Let $2 k \leq r-1$ and $1 \leq q \leq r+1$. Then, the following estimate

$$
\begin{aligned}
\left\|\theta_{k}\right\|_{L^{\infty}\left(L^{2}\right)}+\alpha_{0}\left\|\theta_{k}\right\|_{L^{2}\left(H^{1}\right)} & \leq C(\max (q, 2 k+3), k+1) h^{q+\min (2 k+1, r-1)} \\
& +C\left(h^{2(q-1)}+h^{2 q-d / 2}\right)
\end{aligned}
$$

holds.

Proof. Choose $v=\theta_{k}$ in (5.4) and then, we obtain

$$
\begin{aligned}
\frac{1}{2} \frac{d}{d t}\left\|\theta_{k}\right\|^{2}+B\left(u, u ; \theta_{k}, \theta_{k}\right)= & \left(\frac{\partial}{\partial t} z_{k}, \theta_{k}\right)+\lambda\left\|\theta_{k}\right\|^{2}-\lambda\left(z_{k}, \theta_{k}\right) \\
& +\left(\mathbb{R}_{1}(e, \nabla e), \nabla \theta_{k}\right)+\left(\mathbb{R}_{2}(e, \nabla e), \theta_{k}\right) .
\end{aligned}
$$

A use of the coercivity of $B(u, \nabla u ; \cdot, \cdot)$ with the Youngs inequality and the kickback argument yields

$$
\frac{d}{d t}\left\|\theta_{k}\right\|^{2}+\alpha_{0}\left\|\theta_{k}\right\|_{1}^{2} \leq C\left(\left\|\frac{\partial}{\partial t} z_{k}\right\|_{-1}^{2}+\left\|\mathbb{R}_{1}(e, \nabla e)\right\|_{L^{2}}^{2}+\left\|\mathbb{R}_{2}(e, \nabla e)\right\|_{H^{-1}}^{2}\right)+C\left\|\theta_{k}\right\|^{2} .
$$

Integrating with respect to $t$ and using the estimate (5.2) for $k=1, j=k, s=1$, an application of Gronwall's inequality with estimates (4.20) and (4.21) yields the desired results. This concludes the rest of the proof. 
Remark 5.1 From the above theorem that is, Theorem 5.2, it is observed for $d=1,2,3$ with $\min (2 k+1, r-1)$ as $r-1$ and $q=r+1$ that super-convergence result $\left\|\theta_{k}(t)\right\|=O\left(h^{2 r}\right)$ holds for $r \geq 2$.

As a consequence, error estimates in Sobolev spaces of negative index are easily derive, which are given in the form of a corollary.

Corollary 5.1 Let $2 k \leq r-1,1 \leq q \leq r+1$ and $U(0)$ be defined by (5.3). Then, there holds for $0 \leq s \leq \min (2 k+1, r-1)$

$$
\|(U-u)\|_{L^{\infty}\left(H^{-s}(\Omega)\right)} \leq C(\max (q, 2 k+3), k+1) h^{q+s}
$$

Proof. Since

$$
U-u:=-\theta_{k}+\eta+\sum_{j=1}^{k} z_{j},
$$

a use of estimate (5.5) with estimates (3.19) and (5.2) concludes the result. This completes the proof.

\section{Super Convergence Result for the case of Single Space Variable.}

Consider the case $\Omega=(0,1):=I$ with the finite element subspace being piecewise polynomial functions of degree $r$. Let $\Pi_{h}=\left\{x_{0}, x_{1}, \ldots, x_{N_{h}}\right\}, 0=x_{0}<x_{1}<\ldots<x_{N_{h}}=1$ with $h_{i}=$ $x_{i}-x_{i-1}$ and $\max _{1 \leq i \leq N_{h}} h_{i}=h$. Further, assume that the partition is quasi-uniform, that is, $\max _{1 \leq i, j \leq N_{h}}\left(\frac{h_{j}}{h_{i}}\right) \leq C$, where the constant $C$ is independent of $h$. Assume that $V_{h}^{0}$ consists of $H_{0}^{1}(\Omega)$ functions of $I$ whose restrictions on $I_{j}$ 's are polynomials of degree atmost $r$, where $I_{j}=\left(x_{j-1}, x_{j}\right)$. At each knot $x_{i}, 1 \leq i \leq N_{h}-1$, the element of $V_{h}^{0}$ will be assume to be $C^{p_{i}}$ functions, where $0 \leq p_{i} \leq r$, and $p_{0}=p_{N_{h}}=0$. However, at any knot at which the superconvergence is to take place, the smoothness constraint on $V_{h}^{0}$ must be restricted to continuity only. The properties (3.1) and (3.2) hold true for a such a choice of $V_{h}^{0}$.

Following the procedure, developed by Douglas et al. [13], we now establish the knot superconvergence result of this section.

Let $\bar{x} \in I$ be a knot in each of the partition, that is, for each $h$, there is $i(h)$ such that $\bar{x}=x_{i(h)}$. Further, let $p_{i(h)}=0$. Denote the space $\tilde{H}^{s}$ by

$$
\left\{u:\left.u\right|_{(0, \bar{x})} \in H^{s}((0, \bar{x})),\left.u\right|_{(\bar{x}, 1)} \in H^{s}((\bar{x}, 1))\right\} \times \mathbb{R}
$$

and it's norm be given by

$$
|\|(u, \beta)\||^{2}=\|u\|_{H^{s}((0, \bar{x}))}^{2}+\|u\|_{H^{s}((\bar{x}, 1))}^{2}+\beta^{2} .
$$

For any element $(u, \beta)$ and $(v, \gamma) \in \tilde{H}^{0}$, define the inner product by

$$
[(u, \beta),(v, \gamma)]=(u, v)+\beta \gamma
$$


where $(u, v)$ denotes $L^{2}$ inner product.

The space $\tilde{H}^{-s}$, for $s \geq 0$ is defined by duality with respect to the above inner product, see [2]. For $s \geq 0$ and $z \in H^{1}(I)$, the norm on $\tilde{H}^{-s}$ is given by

$$
|\|z\||_{-s}=\sup \left\{\frac{[(z, z(\bar{x})),(u, \beta)]}{|\|(u, \beta)\||_{s}}:|\|(u, \beta)\||_{s} \neq 0\right\}
$$

It is easy to see

$$
|z(\bar{x})| \leq|\| z|||_{-s}, \quad s \geq 0 .
$$

Now we derive an estimate analogous to the one in Theorem 5.1 for the triple norm.

Theorem 6.1 Let $1 \leq q \leq r+1$ and $0 \leq s \leq r-2 j-1$. Then for $0<h<\epsilon$ sufficiently small and $t \in J$,

$$
\left|\left\|\frac{\partial^{k} z_{j}}{\partial t^{k}}\right\|\right|_{-s} \leq C(\max (q, s+2 j+2), j+k) h^{s+q+2 j}
$$

where $z_{j}$ is defined by (5.1).

Proof. For $(\psi, \beta) \in \tilde{H}^{s}$, define $\phi \in H_{0}^{1}$ by

$$
L^{*}(u) \phi=\psi, x \in I \backslash \bar{x},\left.\quad \frac{\partial A}{\partial \xi}\left(u, u_{x}\right) \phi_{x}\right|_{\bar{x}-0} ^{\bar{x}+0}=-\beta .
$$

Then, $B\left(u, u_{x} ; \mu, \phi\right)=[(\mu, \mu(\bar{x})),(\psi, \beta)]$, for $\mu \in H_{0}^{1}(I)$.

By regularity theorem, it follows that

$$
\text { | }\left|( \phi , \phi ( \overline { x } ) ) \left\|\left.\right|_{s+2} \leq C(s+2,0)|\|(\psi, \beta)\||_{s}\right.\right.
$$

Note that $\rho \in W^{s+1, \infty}(I), \quad \mu \in H_{0}^{1}(I)$

$$
\begin{aligned}
\left(\rho \mu_{x}, \phi_{x}\right) & =\left.\rho(\bar{x}) \mu(\bar{x}) \phi_{x}\right|_{\bar{x}-0} ^{\bar{x}+0}-\int_{0}^{\bar{x}} \mu\left(\rho \phi_{x}\right)_{x} d x-\int_{\bar{x}}^{1} \mu\left(\rho \phi_{x}\right)_{x} d x \\
& =\frac{1}{A_{\xi}\left(u(\bar{x}), u_{x}(\bar{x})\right)} \rho(\bar{x}) \mu(\bar{x}) \beta-\int_{0}^{\bar{x}} \mu\left(\rho \phi_{x}\right) d x-\int_{\bar{x}}^{1} \mu\left(\rho \phi_{x}\right)_{x} d x
\end{aligned}
$$

and hence,

$$
\begin{aligned}
\left|\left(\rho \mu_{x}, \phi_{x}\right)\right| & \leq C|\rho(\bar{x}) \mu(\bar{x}) \beta|+\left|\left(\mu,\left(\rho \phi_{x}\right)_{x}\right)\right| \\
& \leq|\|\mu\||_{-s}|\|(\psi, \beta)\||_{s}
\end{aligned}
$$

where the constant $\mathrm{C}$ depends upon $\|\rho\|_{W^{s+1, \infty}}$, but no higher derivatives of $\rho$.

In order to prove the theorem, we use induction in two ways, that is, an outer induction on $j$ and an inner induction on $k$. 
For $j=0$ and $v \in V_{h}^{0}$, it follows from (6.7), the definition of $B$ and its time derivative that

$$
\begin{aligned}
{\left[\left(\frac{\partial^{k} \eta}{\partial t^{k}}, \frac{\partial^{k} \eta}{\partial t^{k}}(\bar{x})\right),(\psi, \beta)\right] } & =\left[\left(\frac{\partial^{k} \eta}{\partial t^{k}}, \frac{\partial^{k} \eta}{\partial t^{k}}(\bar{x})\right),\left(L^{*}(u) \phi, \beta\right)\right] \\
& =\left(\frac{\partial^{k} \eta}{\partial t^{k}}, L^{*}(u) \phi\right)+\frac{\partial^{k} \eta}{\partial t^{k}}(\bar{x}) \beta \\
& =B\left(u, u_{x}, \frac{\partial^{k} \eta}{\partial t^{k}}, \phi\right) \\
& =B\left(u, u_{x}, \frac{\partial^{k} \eta}{\partial t^{k}}, \phi-v\right)+\sum_{i=1}^{k-1} F_{i k}\left(\frac{\partial^{i} \eta}{\partial t^{i}}, \phi-v\right)-\sum_{i=1}^{k-1} F_{i k}\left(\frac{\partial^{i} \eta}{\partial t^{i}}, \phi\right), \quad v \in V_{h}^{0} .
\end{aligned}
$$

Now using (6.9) for this last term on the right hand side, we arrive at

$$
\begin{aligned}
{\left[\left(\frac{\partial^{k} \eta}{\partial t^{k}}, \frac{\partial^{k} \eta}{\partial t^{k}}(\bar{x})\right),(\psi, \beta)\right] } & \leq C\left\|\frac{\partial^{k} \eta}{\partial t^{k}}\right\|_{1} \inf _{v \in V_{h}^{0}}\|\phi-v\|_{1} \\
& +\sum_{i=1}^{k-1} C(1, k-1)\left\|\frac{\partial^{i} \eta}{\partial t^{i}}\right\|_{1} \inf _{v \in V_{h}^{0}}\|\phi-v\|_{1} \\
& +\sum_{i=1}^{k-1} C(s+2, k-i)\left|\left\|\frac{\partial^{i} \eta}{\partial t^{i}}\right\|\right|_{-s} \mid\|(\psi, \beta)\| \|_{s}
\end{aligned}
$$

The assumption that the elements of $V_{h}^{0}$ are only continuous functions and not differentiable at $x=\bar{x}$ implies that

$$
\inf _{v \in V_{h}^{0}}\|\phi-v\|_{1} \leq C h^{s+1} \mid \|\left(\phi, \phi(\bar{x}) \|\left.\right|_{s+2},\right.
$$

and hence,

$$
\begin{aligned}
\left.\left\|\frac{\partial^{k} \eta}{\partial t^{k}}\right\|\right|_{-s} & \leq C(s+2,0)\left\|\frac{\partial^{k} \eta}{\partial t^{k}}\right\|_{1} h^{s+1}+\sum_{i=1}^{k-1} C(s+2, k-i)\left\|\frac{\partial^{i} \eta}{\partial t^{i}}\right\|_{1} h^{s+1} \\
& +\sum_{i=1}^{k-1} C(s+2, k-i)\left\|\frac{\partial^{i} \eta}{\partial t^{i}}\right\|_{-s} .
\end{aligned}
$$

The case for $j=0$ follows from the Theorem 3.1 and induction hypothesis on $k$.

For $j>0$, the proof is quite similar to the above that is for $v \in V_{h}^{0}$

$$
\begin{aligned}
{\left[\left(\frac{\partial^{k} z_{j}}{\partial t^{k}}, \frac{\partial^{k} z_{j}}{\partial t^{k}}(\bar{x})\right),(\psi, \beta)\right] } & =B\left(u, u_{x} ; \frac{\partial^{k} z_{j}}{\partial t^{k}}, \phi\right) \\
& =B\left(u, u_{x} ; \frac{\partial^{k} z_{j}}{\partial t^{k}}, \phi-v\right)+\sum_{i=1}^{k-1} F_{i k}\left(\frac{\partial^{i} z_{j}}{\partial t^{i}}, \phi-v\right) \\
& -\sum_{i=1}^{k-1} F_{i k}\left(\frac{\partial^{i} z_{j}}{\partial t^{i}}, \phi\right)+\left(\frac{d^{k}}{d t^{k}}\left(\frac{\partial z_{j-1}}{\partial t}\right), \phi-v\right) \\
& -\left(\frac{d^{k}}{d t^{k}}\left(\frac{\partial z_{j-1}}{\partial t}\right), \phi\right) .
\end{aligned}
$$


Similar to the case $j=0$, now an appropriate application of (6.7) with (6.8), (6.9) and (6.10) yields

$$
\begin{aligned}
\left.\left\|\frac{\partial^{k} z_{j}}{\partial t^{k}}\right\|\right|_{-s} & \leq C(s+2,0)\left\|\frac{\partial^{k} z_{j}}{\partial t^{k}}\right\|_{1} h^{s+1}+\sum_{i=1}^{k-1} C(s+1, k-i)\left\|\frac{\partial^{i} z_{j}}{\partial t^{i}}\right\|_{1} h^{s+1} \\
& +\sum_{i=1}^{k-1} C(s+2, k-i)\left|\left\|\frac{\partial^{i} z_{j}}{\partial t^{i}}\right\|\right|_{-s}+C(s+1,0)\left|\left\|\frac{\partial^{k+1}}{\partial t^{k+1}} z_{j-1}\right\|\right|_{-1} h^{s+1} \\
& +C(s+1,0)\left|\left\|\frac{\partial^{k+1}}{\partial t^{k+1}} z_{j-1}\right\|\right|_{-s-2} .
\end{aligned}
$$

Now the conclusion of the theorem follows from Theorem 5.1 and by Induction.

From (6.5) and (6.6), it follows that

$$
\left|z_{j}(\bar{x}, t)\right| \leq C(\max (q, s+2 j+1), j) h^{q+s+2 j}, \quad j=0,1,2, \ldots
$$

The next Theorem constitutes the main result of the present work.

Theorem 6.2 Let $1 \leq q \leq r+1$ and $k$ be an integer satisfying $0 \leq k \leq\left[\frac{r-1}{2}\right]$. Let $u$ be $a$ sufficiently regular solution of (1.1) and $U$ the Galerkin approximate solution is given by (3.4) with $U(0)=\tilde{u}(0)+z_{1}(0)+z_{2}(0)+\ldots+z_{k}(0)$, with $z_{k}^{\prime}$ is defined as in (5.3). Then, there is a positive constant $C$ such that

$$
|(u-U)(\bar{x}, t)| \leq \begin{cases}C(\max (q, 2 k+3, k+1)) h^{-\frac{1}{2}}\left(h^{q+2 k}+h^{2 q-2}\right) ; & 2 k \leq r-2 \\ C(\max (q, 2 k+3, k+2)) h^{-\frac{1}{2}}\left(h^{q+2 k+1}+h^{2 q-2}\right) ; & 2 k \leq r-1\end{cases}
$$

where $\bar{x}=x_{i}(h)$ is a knot at which the smoothness of $V_{h}^{0}$ reduces to continuity.

Proof. Writing $u-U$ in the form

$$
(u-U)(\bar{x}, t)=\theta_{k}(\bar{x}, t)-\sum_{j=0}^{k} z_{j}(\bar{x}, t)
$$

and using inverse property (3.2), for the finite dimensional space $V_{h}^{0}$, we obtain

$$
\left|\theta_{k}(\bar{x}, t)\right| \leq C h^{-\frac{1}{2}}\left\|\theta_{k}(\cdot, t)\right\| .
$$

On combining (6.13), (6.16) and (5.5), we arrive at the required result and this concludes the rest of the proof.

Corollary 6.1 For $k=\left[\frac{r-1}{2}\right]$ and $q=r+1$

$$
|(u-U)(\bar{x}, t)| \leq \begin{cases}C\left(r+1, \frac{1}{2}(r+1)\right) h^{2 r-\frac{1}{2}}, & \text { for odd } r \\ C\left(r+1, \frac{1}{2}(r+1)\right) h^{2 r-\frac{1}{2}}, & \text { for even } r .\end{cases}
$$

Remark 6.1 There is a deterioration of order $\frac{1}{2}$ in the superconvergence as compared to the order in Arnold and Douglas [2]. However, in stead of using (6.16), one can exploit the superconvergence property of $\left\|\theta_{k}\right\|_{1}$ norm, but we still loose half power in the superconvergence result. Therefore, we believe that this is due to the gradient term present in the operator $A$ in the equation (1.1). 


\section{Conclusion}

Based on Taylor's expansion, an elliptic projection is developed for the steady state linearized problem and related error estimates are derived. Optimal error estimates in both $L^{\infty}\left(L^{2}\right)$ and $L^{\infty}\left(H^{1}\right)$-norms are proved for the quasilinear parabolic problems with nonlinearity depending on gradient by employing the Gronwall type result, which hold true for $d=1,2,3$. These results improve upon the earlier results of [3]. A use of quasi-project technique yields a superconvergence result for the error between the Galerkin approximation and quasi-projection and as a consequence, negative norm estimates between the exact solution and semidiscrete Galerkin approximation are established. It is observed that the Bramble-Schatz post processing, see [6] combined with negative norm estimate may provide superconvergence results, which can be achieved provided some estimates of the difference quotients are shown to be true for this nonlinear problem. In a single space variable, superconvergence results at the nodal points are derived. Developing higher order time discretization method for the completely discrete schemes combined with quasi-projection tool shall be more challenging and will be a part of our future endevour.

Acknowledgement. This research of the second author is supported by Chiangmai University and the Centre of Excellence in Mathematics, The Commmision on Higher Education, Thailand.

\section{References}

[1] S. Agmon, Lectures on Elliptic Boundary Value Problems, Van Nostrand, Princeton,1965.

[2] D.N. Arnold and J. Douglas,Jr., Superconvergence of the Galerkin Approximation of a Quasilinear Parabolic Equation in a Single Space Variable, CALCOLO, XVI (1979), pp. 345-369.

[3] O. Axelsson, Error Estimates for Galerkin Methods for Quasilinear Parabolic and Elliptic Differential Equations in Divergence Form, Numer. Math., 28 (1977), pp. 1-14.

[4] C. Bi and Y. Lin, Discontnuous Galerkin method for monotone nonlinear elliptic problems, IJNAM, 9 (2012), pp. 999-1024.

[5] J.H. Bramble, A.H. Schatz, V. Thomée and L.B. Wahlbin, Some convergence estimates for semidiscrete Galerkin type approximations for parabolic equations, SIAM J. Numer. Anal., 14 ( 1977), pp. 218-241.

[6] J.H. Bramble and A.H. Schatz, Higher order local accuracy by averaging in the finite element method, Math. Comp., 31 (1977), pp. 94-111.

[7] S.C. Brenner and L. R. Scott, The Mathematical Theory of Finite Element Methods, third ed., Texts in Applied Mathematics, Vol. 15, Springer, New York, 2008.

[8] C.D. Boor, On Local spline approximation by moments, J. Math. Mech., 17 (1967/68), pp.729735 . 
[9] W. Cao and Z. Zhang, Some recent developments in superconvergence of discontinuous Galerkin methods for time-dependent partial differential equations, J. Sci. Comput., 77 (2018), pp.14021423.

[10] S.S. Chow, Finite element error estimates for non-linear elliptic equations of monotone type, Numer. Math., 54 (1989), pp. 373-393.

[11] J. Douglas,Jr.,T.Dupont and J.Serrin, Uniqueness and comparison theorm for nonlinear elliptic equations in divergence form, Arch.,Rat. Mech.Anal., 42 (1971),pp. 157-168

[12] J. Douglas,Jr.,T. Dupont and L.Walbin, Optimal $L^{\infty}$ Error estimates for Galerkin approximation to solution of two-point boundary value problems, Math. Comp., 29 (1975), pp.475 $-483$

[13] J. Douglas, Jr., T. Dupont and M. F. Wheeler, A quasi-projection analysis of Galerkin methods of parabolic and hyperbolic equations, Math.Comp., 32 (1978), pp.345 -362.

[14] M. Feistauer and A. Zenisek, Finite element solution of nonlinear elliptic problems, Numer. Math., 50 (1987), pp. 451-475.

[15] M. Feistauer and A. Zenisek, Compactness method in the finite element theory of nonlinear elliptic problems, Numer. Math., 52 (1988), pp. 147-163.

[16] T. Gudi, N. Nataraj, A.K. Pani, hp-discontinuous Galerkin methods for strongly nonlinear elliptic boundary value problems, Numer. Math.,109 (2008), pp. 233-268.

[17] P. Houston, J. Robson, E. S uli, Discontinuous Galerkin finite element approximation of quasilinear elliptic boundary value problems I: The scalar case, IMA J. Numer. Anal., 25, (2005), pp. 726-749.

[18] J. Kacur, Solution to strongly nonlinear parabolic problems by a linear approximation scheme, IMA J.Numer. Anal.,19 (1999), pp. 119-145.

[19] M.Y. Kim, F.A. Milner and E.-J. Park, Some observations on mixed methods for fully nonlinear parabolic problems in divergence form, Appl. Math. Lett., 9 (1996), pp. 78-81.

[20] M. Krizek, and P. Neittaanm aki, On superconvergence techniques, Acta Applicandae Mathematicae, 9 (1987), pp.175-198.

[21] M. Krizek, P. Neittaanm aki, and R. Stenberg, (eds.),Finite Element Methods: Superconvergence, Postprocessing, and A Posteriori Estimates, Lecture Notes in Pure and Applied Mathematics Series, Vol. 196, Marcel Dekker, Inc., New York (1997).

[22] S. Kundu, A. K. Pani, M. Khebchareon, On Kirchoff's model of parabolic type, Numer. Func. Anal.Opt., 37 (2016), pp.719-752. 
[23] O.A. Ladyzenskaja,V.A. Solonnikov and N.N. Ural'ceva, Linear Quasilinear Equation Of Parabolic Type,American Math.Soc.,Providence,Rhode Island, 1968.

[24] L. Jones and A. K. Pani On superconvergence results and negative norm estimates for a unidimensional single phase Stefan problem, Numer Funct. Anal. Optim., 16 (1995), pp. 153-175.

[25] F.A. Milner and E.-J. Park, A mixed finite element method for a strongly nonlinear second-order elliptic problem, Math. Comp., 64 (1995), pp. 973-988.

[26] J.M. Ortega and W.C Rheinboldt, Iterative Solution of NonLinear Equation in Several Variable, Academic Press, New York-London,1970.

[27] A.K. Pani and R. K. Sinha, On Superconvergence results and negative norm estimates for parabolic integro-differential equations, J. Integral Equ., 8 (1996), pp. 65-98.

[28] E.J. Park, A primal hybrid finite element method for a strongly nonlinear second-order elliptic problem, Numer. Meth. PDE. 11 (1995), pp. 61-75.

[29] E.J. Park, Mixed Finite element methods for generalized Forchheimer flow in porous media, Numer. Meth. PDE. 21 (2005), pp. 213-228.

[30] N. Sharma, M. Khebchareon and A. K. Pani, A priori error estimates of expanded mixed FEM for Kirchhoff type parabolic equation, Numer. Algo. 83 (2020), pp. 125-147.

[31] V. Thomée, Negative norm estimates and superconvergence in Galerkin Methods for Parabolic Problems, Math.Comp.,34 (1980), pp. 93-113.

[32] N.S. Trudinger, On Comparision Principle for Quasilinear Divergence Structure Equation, Arch.Rat.Mech.Anal. 57 (1974), pp. 128-133.

[33] L. Wahlbin, Superconvergence in finite element methods, Lecture Notes in Mathematics, vol. 1605, Springer Verlag, Berlin, 1995.

[34] M.F. Wheeler, A priori $L^{2}$-Error Estimates for Galerkin Approximation to Parabolic Partial Differential Equations, SIAM J.Numer.Anal. 10 (1973), pp.723-758.

[35] S. Yadav and A. K. Pani, Superconvergence of a class of expanded discontinuous Galerkin methods for fully nonlinear elliptic problems in divergence form, J. Comp. Appl. Math. 333 (2018), pp. 215-234. 